\title{
Cadena productiva de lana de oveja en el sector textil y de confecciones
}

\section{RESUMEN}

El Perú presenta producción de ovinos en todos los departamentos del país. La fibra natural obtenida de las ovejas permite obtener diversas modalidades textiles $y$ de confecciones. Esto no se refleja significativamente en una presencia comercial dichos productos. En el mercado interno los productos derivados de la lana de oveja se orientan básicamente a prendas de origen artesanal, como chompas, bufandas; mientras que en el mercado externo la presencia de exportaciones es muy reducida. El enfoque de cadena productiva permite una visión sistémica del problema y busca encadenar los diferentes elementos que la componen. La cadena productiva de la lana de oveja presenta tres fases marcadas: en el sector primario, se ubican los productores y acopiadores, así como la atención sanitaria, Una vez esquilada, la lana pasa a procesos característicos del sector industrial, como son el lavado, cardado, semi peinado y peinado, de modo tal que se tiene hilos o telas en base a lana de oveja, los cuales pueden pasar por la manufactura y convertirse en productos del rubro de textiles y confecciones. Finalmente, intervienen los comercializadores, tanto para el mercado interno como para la exportación.

Palabras clave: Cadena productiva, lana de oveja, textiles y confecciones

Productive Chain of THE WOOL Of EWE IN THE TEXTILE SECTOR AND CONFECTIONS

\section{ABSTRACT}

Peru presents production of sheep in all departments. The natural fiber obtained from sheep allows for various textile and garment patterns. This is not reflected significantly in a commercial presence such products. In the domestic market for products derived from sheep's wool garments are geared primarily to artisanal origin as sweaters, scarves, whereas in the presence of foreign market exports is very small. The value chain approach allows a systemic view of the problem and seek to chain the different elements that compose it. The production chain wool marked three phases: in the primary sector, there are the producers and assemblers, as well as health care, Once sheared, the wool becomes characteristic of the industry processes, such as washing, carded, combed and brushed semi, so you have yarn, fabric based on wool, which can pass through the manufacturing and become the object of textile products and apparel. Finally, marketers involved, both for the domestic market and for export.

Key words: Productive chain, wool of sheep, textiles and confections

\section{INTRODUCCIÓN}

El 2009 fue declarado por la FAO (Organización de las Naciones Unidas para la Agricultura) como el "Año internacional de las fibras naturales", confirmando una tendencia mundial creciente de interés por los productos orgánicos y naturales. La lana de oveja, al igual que el algodón, el cáñamo, entre otros, forma parte de esta variedad de fibras que contribuyen al desarrollo de los textiles y las confecciones.

La industria de textiles, confecciones y cuero están contenidas en el sector manufacturero y en los últimos años ha presentado una importante cuota en la estructura del Producto Bruto Interno (PBI) global. Según cifras del Instituto Nacional de Estadística e Informática (INEI) la participación de la industria textil, confecciones y del cueros en la estructura del PBI global fue de 2,2\% en el 2006, sin embargo, el nivel más alto se registró en el año 2004 , donde la participación de dicho sector ascendió a 2,5\%. La reciente crisis mundial ha afectado severamente al sector en este último año.

El sector textil representa para muchos países sudamericanos un importante aporte de divisas por su creciente actividad exportadora.

Según el Boletín Sectorial de Textiles y Confecciones del Ministerio de Trabajo y Promoción del Empleo del Perú (2004), "el sector de Textiles y Confecciones es un sector altamente competitivo, competencia que se relaciona directamente con el nivel de inversión. Para poder ser una industria altamente competitiva, se requiere de mejoras tecnológicas incorporadas en diferentes tipos de maquinaria y equipo, cuya complejidad en su uso dependerá de las características de la empresa (las más grandes cuentan por lo general con maquinarias mucho más modernas,a diferencia de las pequeñas cuya producción se orienta a la pequeña escala)".

Según un informe del Ministerio de Trabajo y Promoción del Empleo, respecto a la generación de empleo por sectores empresariales, (2007), la industria textil genera encadenamientos hacia atrás con industrias proveedoras de materia prima, y encadenamientos hacia adelante con industrias distribuidoras

\footnotetext{
Magíster en Marketing y Turismo. Docente Auxiliar del Departamento de Ingeniería de Sistemas
} e Informática de la UNMSM. E-mail: otinocog@gmail.com 
y de servicios complementarios a sus productos. Esto ha generado que las mayores exportaciones de textiles provoquen un incremento en la demanda de trabajadores por parte de las empresas de Lima Metropolitana y del interior del país, especialmente las ubicadas en las ciudades de Arequipa y Chincha.

La lana de oveja forma parte de la visión global de la cadena productiva que involucra textiles y confecciones, pero, a diferencia del algodón y de la lana de alpaca, no ha recibido la atención correspondiente que la involucre positivamente es este importante esfuerzo. Tal es así que en las estadísticas de exportaciones textiles, la lana de oveja presenta una mínima participación.

\section{CUADRO 1: SECTOR TEXTIL CONFECCIONES POR MATERIALES (MILLONES US\$)}

\begin{tabular}{|l|c|c|}
\hline & \multicolumn{2}{|c|}{ Abril } \\
\hline Material & 2008 & 2009 \\
\hline Algodón & 135,1 & 72,4 \\
\hline Sintético & 22,3 & 13,6 \\
\hline Alpaca & 7,5 & 5 \\
\hline Otros & 6,9 & 8 \\
\hline
\end{tabular}

Fuente: SUNAT.

La lana de oveja es una fibra natural que se extrae del vellón de las ovejas, variando sus características según la raza del animal. El Ministerio de Agricultura (Minag, 2007) a través de la Dirección General de Promoción Agraria (DGPA) ha sistematizado las características principales de la lana de oveja y promueve las cadenas productivas en productos estratégicos como el algodón.

En su portal correspondiente, el MINAG, a través del portal denominado cadena productiva promueve sistemáticamente el trabajo con cadenas productivas, habiendo publicado virtualmente una Guía Metodológica para tal efecto, en donde se define la cadena productiva como "un sistema que agrupa a los actores económicos interrelacionados por el mercado con participación articulada en actividades que generan valor, alrededor de un bien o servicio". En este proceso se incluyen las fases de provisión de insumos producción, conservación y transformación. (pág. 2)

Los productores agroindustriales de lana, consideran que obtener materia prima de calidad, confiable y segura es una exigencia que impone el mercado, situación que cobra mayor importancia en el marco de los tratados de Libre Comercio con los Estados Unidos de Norte América, China y la Unión
Europea. El mercado internacional demanda fibras sanas, con adecuado desarrollo a lo largo de la mecha, sin debilidades en su crecimiento, con baja variabilidad en sus características y reducidos niveles de contaminación.

\section{MARCO TEÓRICO}

\section{La lana ovina}

Las fibras naturales son sustancias muy alargadas producidas por plantas y animales, que se pueden hilar para obtener hebras, hilos o cordelería. Según información de la FAO, con motivo del año internacional de las fibras naturales, se producen alrededor de 30 millones de toneladas de fibras naturales al año en todo el mundo. Las fibras naturales son un elemento importante del vestido, la tapicería y otros textiles de consumo.

La lana es una fibra textil formada en los folículos de la piel del ovino que integra el vellón del animal. Constituye una fibra suave y rizada, que en forma de vellón recubre el cuerpo de las ovejas. Está formada a base de la proteína llamada queratina, en torno al $20-25 \%$ de proporción total.

Cada fibra es segregada en un folículo piloso y consta de una cubierta externa escamosa (lo que provoca el enfieltrado) que repele el agua, una porción cortical y otra medular (que absorbe la humedad). Varía entre 12 y 120 micras de diámetro, según la raza del animal productor y la región de su cuerpo, y entre 20 y 350 mm de longitud.

Los usos de la lana están en relación directa con una gama de diámetros que la hacen utilizable en ropa (industria textil). Dos tercios de la lana van a la manufactura de prendas (telas, chompas, abrigos, etc) Un tercio se destina a la manufactura de sábanas antiestáticas o alfombras antirruido.

En la industria de la construcción se utilizan hojas aglomeradas de lana gruesa para aislamiento térmico y/o acústico.

\section{Cadena productiva}

El concepto de la cadena productiva fue desarrollado como instrumento de visión sistémica. Parte de la premisa que la producción de bienes se puede representar como un sistema, donde flujos de materiales, de capital y de información conectan a los diversos agentes de la cadena que buscan proveer un mercado consumidor final de los productos del sistema. (Gomes de Castro et al., 2002) 


\section{FICHA TÉCNICA DE LA LANA DE OVEJA}

\begin{tabular}{|l|l|}
\hline Origen & Es una fibra natural que se extrae del vellón de las ovejas, mediante esquilado. \\
\hline Aspecto & La fibra de lana es rizada y ondulada y se presenta recubierta de escamas. \\
\hline Longitud & $\begin{array}{l}\text { Tiene una longitud aparente, sin perder el rizo natural, la cual es distinta a la longitud real, cuando } \\
\text { está extendida. A mayor longitud de esta fibra se registra mayor diámetro. }\end{array}$ \\
\hline Propiedades & $\begin{array}{l}\text { Tomando como referencia su procedencia y diámetro, se clasifican en extra, extrafina, fina, } \\
\text { entrefina, ordinaria, basta y muy basta. }\end{array}$ \\
\hline Inconvenientes & $\begin{array}{l}\text { Es resistente, elástica y flexible. Su capacidad de protección térmica le configura un adecuado } \\
\text { boder aislante. Cuenta con gran capacidad de absorción de humedad y se arruga poco. Registra } \\
\text { buena elasticidad, es antiinflamable y no se funde. }\end{array}$ \\
\hline Tipos & $\begin{array}{l}\text { Responde mal a los roces, en estado húmedo tiende a formar "bolas" y a enfieltrarse. Las polillas le } \\
\text { cruce, Cheviots, Shelland, entre otros. En el Perú tiene significativa presencia la raza "criolla". }\end{array}$ \\
\hline
\end{tabular}

Fuente: Elaboración propia en base a información de Wilder Huamán.

CUADRO 2. POBLACIÓN DE GANADO OVINO, SEGÚN UNIDAD AGRARIA, 2001-2007

\begin{tabular}{|l|r|r|r|r|r|r|r|}
\hline \multicolumn{1}{|c|}{ Unidad agraria } & $\mathbf{2 0 0 1}$ & $\mathbf{2 0 0 2}$ & $\mathbf{2 0 0 3} \mathbf{P I}$ & $\mathbf{2 0 0 4} \mathbf{P I}$ & $\mathbf{2 0 0 5} \mathbf{P I}$ & $\mathbf{2 0 0 6} \mathbf{P I}$ & $\mathbf{2 0 0 7} \mathbf{P I}$ \\
\hline La Libertad & 539.157 & 535.702 & 503.783 & 454.298 & 402.413 & 420.429 & 413.495 \\
\hline Áncash & 922.779 & 827.885 & 827.470 & 825.468 & 847.298 & 807.840 & 793.399 \\
\hline Lima & 350.598 & 365.837 & 352.714 & 352.304 & 352.067 & 340.000 & 324.238 \\
\hline Arequipa & 294.012 & 297.300 & 295.343 & 288.827 & 286.680 & 269.022 & 266.519 \\
\hline Cajamarca & 328.382 & 332.983 & 372.500 & 373.100 & 375.069 & 391.715 & 426.561 \\
\hline Huánuco & $\mathbf{1 . 2 3 4 . 4 1 3}$ & $\mathbf{1 . 1 1 3 . 9 1 7}$ & $\mathbf{1 . 1 4 7 . 6 0 0}$ & $\mathbf{1 . 1 4 8 . 1 0 0}$ & $\mathbf{1 . 1 4 7 . 6 5 7}$ & $\mathbf{1 . 1 7 0 . 8 6 6}$ & $\mathbf{1 . 0 5 4 . 5 0 4}$ \\
\hline Pasco & 916.030 & 925.190 & 930.346 & 939.694 & 947.800 & 934.944 & 950.105 \\
\hline Junín & $\mathbf{1 . 2 9 9 . 0 0 0}$ & $\mathbf{1 . 2 7 0 . 8 0 0}$ & $\mathbf{1 . 2 0 3 . 4 7 0}$ & $\mathbf{1 . 2 0 3 . 6 0 0}$ & $\mathbf{1 . 2 1 4 . 2 1 0}$ & $\mathbf{1 . 2 1 6 . 0 0 0}$ & $\mathbf{1 . 2 1 4 . 2 1 0}$ \\
\hline Huancavelica & 962.500 & 970.243 & 964.800 & 978.452 & 993.165 & 976.712 & 975.777 \\
\hline Ayacucho & 907.974 & 921.394 & 891.036 & 859.133 & 905.080 & 820.966 & 846.254 \\
\hline Apurímac & 476.510 & 476.674 & 476.674 & 476.240 & 467.210 & 504.320 & 518.531 \\
\hline Cusco & $\mathbf{2 . 1 8 7 . 6 2 1}$ & $\mathbf{2 . 2 7 5 . 1 2 5}$ & $\mathbf{2 . 3 8 8 . 0 0 0}$ & $\mathbf{2 . 3 8 9 . 0 0 0}$ & $\mathbf{2 . 3 8 8 . 8 8 2}$ & $\mathbf{2 . 3 8 5 . 0 0 0}$ & $\mathbf{2 . 3 0 0 . 0 0 0}$ \\
\hline Puno & $\mathbf{3 . 8 6 0 . 8 1 0}$ & $\mathbf{3 . 9 2 4 . 1 6 0}$ & $\mathbf{3 . 8 9 6 . 4 0 0}$ & $\mathbf{3 . 8 9 6 . 4 5 0}$ & $\mathbf{3 . 9 3 2 . 0 7 0}$ & $\mathbf{3 . 8 7 0 . 2 2 0}$ & $\mathbf{3 . 9 1 7 . 9 2 0}$ \\
\hline
\end{tabular}

Fuente: Ministerio de Agricultura - Dirección General de Información Agraria. 2008.

Para la teoría de los sistemas, el todo (o el sistema) es el producto de las partes interconectadas, cuyo conocimiento y estudio debe suceder siempre relacionando el funcionamiento de estas partes en lo referente al todo.

El enfoque sistémico se puede expresar en el modelo de cadenas productivas, cuando se trata de macro procesos productivos de cualquier naturaleza. Este abordaje, inicialmente difundido en el sector agrícola, fue utilizado con éxito en otros sectores productivos como el sector industrial. Tales registros apuntan hacia la emergencia de un enfoque metodológico con gran capacidad de extrapolación y amplio uso. (Gomes de Castro et al., 2002).

Entidades peruanas, estatales y privadas, han mostrado interés en este enfoque y sus aplicaciones prácticas.

Para la ONG MINKAPERÚ: "la Cadena Productiva es un sistema conformado por la interacción entre diversos participantes directamente involucrados en la producción de productos y servicios desde la producción hasta el consumo. La cadena va más allá del territorio y su estructura y formas de coordinación varían dependiendo del sector, del territorio y del tipo de cadena. La forma como las pequeñas empresas pueden insertarse en una cadena depende de sus capacidades para responder a las exigencias de los compradores. Estas exigencias son estándares de calidad, laborales, medioambientales etc. que permiten o restringen el acceso a las cadenas. (San Martín, 2008). 
El Ministerio de Agricultura define las cadenas productivas como el "conjunto de agentes económicos que participan directamente en la producción, en la transformación y en el traslado hacia el mercado de un mismo producto agropecuario".

El proyecto de implementación del Corredor Cusco-Puno (2006), en el sur peruano, reporta una primera aproximación a la importancia de la lana de ovinos en las políticas de integración económica. Por un lado, se encuentran los productos de los ovinos de raza, Corriedale y Junín, que se caracterizan por vellones de finura media (24-31 micras), buena longitud $(8-11 \mathrm{~cm})$, alto rendimiento al lavado (65-70\%) y buen grado de resistencia. Este producto es destinado en su totalidad a la industria textil nacional. Los vellones son clasificados de acuerdo al Sistema Peruano, luego prensados y enfardados en telas de yute.

Por otro lado, los vellones de ovinos criollos presentan características de finura, longitud muy variables, así como un bajo rendimiento al lavado. Tienen menores precios que las lanas de Corriedale y Junín, y son utilizadas en la elaboración de productos artesanales.

En un estudio realizado en Argentina por la Agencia Nacional de Promoción de Ciencia y Tecnología para la zona ganadera de Chubut y Jujuy (2006), respecto al proceso productivo, se reportan "problemas asociados a la contaminación por fibras teñidas por heces y orina (puntas amarillas), fibras pigmentadas (de origen genético) y fibras con impurezas total o parcialmente (pelos, chillas, kemps, etc.) resultan una complicación en el proceso de teñido industrial para la obtención de tejidos de colores claros o pastel".

Todo esto indica la necesidad de una visión integral, sistémica, del proceso de la lana proveniente de ovinos, encadenando a los diferentes actores que intervienen en ella, desde la obtención de la materia prima (lana) hasta la comercialización de los productos textiles.

\section{Cadena productiva de la lana de oveja}

Definimos la cadena productiva de la lana de oveja como la interacción de los diferentes agentes económicos que participan directamente en la producción, transformación y comercialización de la lana de ovinos; forman parte complementaria de ella las organizaciones educativas, financieras y de apoyo técnico, así como aquellas que desarrollan investigación y/o capacitación en este aspecto, en la bús- queda de mayor competitividad y de mejor redistribución de los ingresos en el sector.

Los principales actores de la cadena productiva de la lana de oveja son los productores de ovinos (pequeños, medianos y grandes), los acopiadores de lana, aquellos que transforman la fibra en insumo para el hilado (artesanal o industrial) y los comercializadores, tanto internos como externos.

La cadena productiva de la lana de oveja en el Perú tiene como escenario un corredor económico conformado por agentes, materias primas e insumos, acoplados de modo directo o indirecto, en los procesos de producción, intermediación, transformación a escala industrial y artesanal y comercialización de la fibra y otros productos de dichos ovinos. La zona de mayor incidencia es la altoandina, como puede apreciarse en el Cuadro 2:

Otros componentes colaterales y acompañantes de esta cadena, son la investigación y desarrollo básico, desarrollado por entidades universitarias (IVITA San Marcos, UNALM) o empresas privadas del sector; servicios de formación, capacitación, investigación y desarrollo tecnológico textil (SENATI); sistemas de crédito y financiamiento; información, inteligencia de mercados y marketing, principalmente.

La cadena de valor y la cadena productiva de la lana de ovinos se desarrollan a partir del sector primario (agropecuario), pasando al sector industrial y luego al de comercialización, tal como se detalla en el Diagrama 1.

El productor: en unos casos es el criador de los ovinos que al mismo tiempo es su propietario y quien lo pastorea, cuida, maneja y atiende directamente.

Los intermediarios: son los agentes de la cadena que intervienen en la compra, acopio y venta de la fibra grasienta del productor. Pueden ser minoristas o mayoristas.

Los industriales textiles: son las empresas transformadoras de la fibra grasienta en productos textiles: hilos, telas y confecciones.

Los artesanos textiles: son fabricantes de confecciones que utilizan tecnología artesanal en base a fibras procesadas por la industria textil y/o por los hilanderos artesanales que son quienes fabrican hilos con maquinaria artesanal. En la última déca- 
DIAGRAMA 1: CADENA PRODUCTIVA DE LA LANA DE OVEJA

\begin{tabular}{|c|c|c|c|c|}
\hline \multicolumn{2}{|c|}{ Sector Primario } & \multicolumn{2}{|c|}{ Sector Industrial } & Comercialización \\
\hline \begin{tabular}{|l|} 
PRODUCCIÓN Y \\
REPRODUCCIÓN \\
Razas \\
Alimentación \\
Engorde \\
\end{tabular} & \begin{tabular}{|l} 
ESQUILADO \\
\\
Tipos \\
Australiano \\
Tally - Hi \\
\end{tabular} & CARDADO & $\begin{array}{l}\text { De lana cardada } \\
\text { De lana peinada } \\
\text { Enconado }\end{array}$ & $\begin{array}{l}\text { MERCADO INTERNO } \\
\text { Distribución / consumo } \\
\text { Acopiador (lana) } \\
\text { Confección / Diseño } \\
\text { Comercialización } \\
\text { Artesanía } \\
\end{array}$ \\
\hline & $\begin{array}{l}\text { Acondicionado } \\
\text { Clasificación }\end{array}$ & SEMI PEINADO & TEJEDURIAS & $\begin{array}{c}\text { EXPORTACIÓN } \\
\text { Exportación con industrias }\end{array}$ \\
\hline \begin{tabular}{|l}
\multicolumn{1}{|c}{ Control S } \\
Enfermedades \\
Vacunas \\
\end{tabular} & anitario & PEINADO & $\begin{array}{l}\text { Preparación hilados } \\
\text { Tejido plano / punto } \\
\text { Terminado }\end{array}$ & $\begin{array}{l}\text { Exportador independiente } \\
\text { Artesania }\end{array}$ \\
\hline
\end{tabular}

da se vienen desarrollando artesanos que utilizan tintes naturales, provenientes de flores y algunos minerales, para el teñido de la lana de oveja.

Los comercializadores: son aquellos entes, naturales o jurídicos, que compran y venden los productos textiles, tanto para el mercado local, regional y nacional, como para exportación, industrial o artesanal.

La materia prima en todo este proceso es la lana de las ovejas. A nivel mundial existen 450 razas de ovejas, cuya crianza está orientada a la producción de carne (Hampshire Down, Southdown, Texel, entre otros), de lana (merino australiano, merino Rambouillet, entre otros) o mixtas (Corriedale, Lincoln, Ideal, entre otros).

En el ande peruano, las ovejas forman parte de la magra economía de los campesinos, de la denominada raza "criolla", en muchas zonas se crian las razas merino.

Una primera consideración en el inicio de la cadena productiva es precisamente la selección de la raza, hecho que las empresas agroindustriales vienen realizando con notable éxito, pero que no es el común denominador en los productores individuales o las organizaciones campesinas.

Asociado a la raza se encuentra el tipo de alimentación (pasturas y complementos alimenticios), los controles y cuidados sanitarios (vacunas, remedios).

El siguiente paso consiste en el esquilado, en el cual las ovejas son aquietadas para despojarlas de su lana. Esto implica, desde el punto de vista agroindustrial, técnicas y equipos depurados, mientras que en el sector comunal responde muchas veces a su cosmovisión y a métodos artesanales.

Una vez obtenida la lana, se procede a su clasificación, atendiendo a los criterios de finura, longitud, resistencia y rendimiento.

Ya en el sector industrial de la cadena productiva, la primera preocupación es el lavado de la lana. El lavado se realiza en forma mecánica en máquinas denominadas lavaderos, que constan de cuatro a cinco tinas de gran capacidad y con mecanismos de arrastre que permiten el avance de la lana dentro de cada tina.

El siguiente proceso es el cardado. Su función es la de paralelizar las hebras de lana y eliminar parte de la materia vegetal que no haya salido en el lavado. Se inicia así el proceso de estiramiento. Luego se procede al semi peinado; en donde el producto cardado pasa a otra máquina llamada Gill o Intersecting con la finalidad de estirar más la lana, paralelizando mejor las hebras y eliminando gran parte de la materia vegetal.

Finalmente se desarrolla el peinado. Su función es terminar de paralelizar y limpiar de materia vegetal a la lana. El producto de este proceso es el TOP. En este proceso se cuenta con un subproducto denominado Bluss o Noils que es lana corta pero limpia, la misma que es usada para la confección de telas no tejidas y afieltrados (para sombrerería). El producto obtenido en la fase anterior pasa a la hilanderia (de lana cardada o lana peinada) o tejeduría (tejido de punto o tejido plano). 


\section{GRÁFICO 1. PERÚ: POBLACIÓN DE OVINOS} POBLACIÓN GANADO OVINO

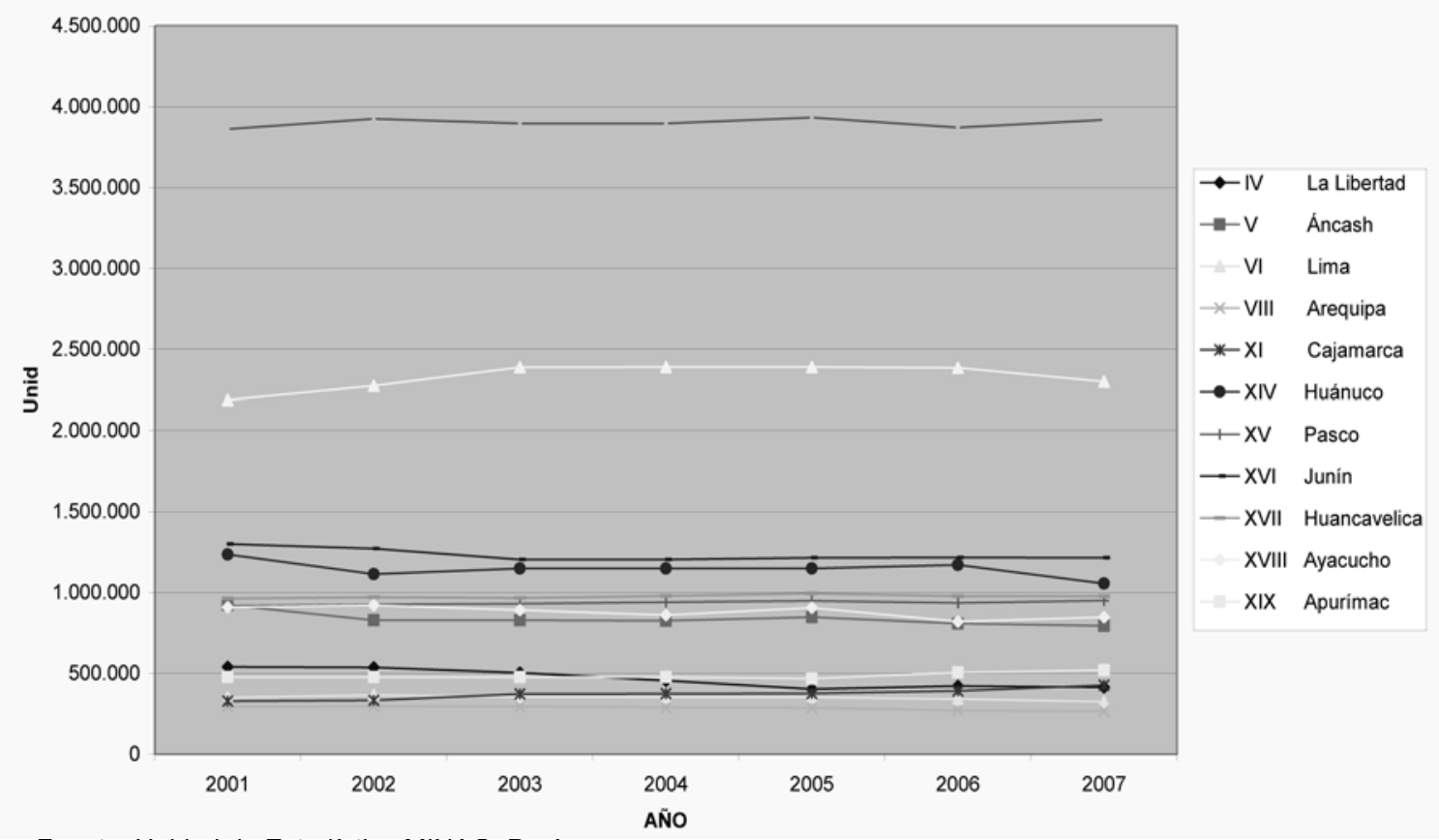

Fuente: Unidad de Estadistica MINAG. Perú.

En el proceso del hilado, se elaboran los hilos de diferentes calidades, mezclas, colores naturales y artificiales. Luego se continúa con el teñido, para presentar la fibra en una amplia gama de colores.

En el ámbito artesanal, destaca el uso del teñido de la lana de oveja con tintes naturales, actividad desarrollada por organizaciones campesinas con asesoramiento de Organizaciones No Gubernamentales (ONG), que en muchos casos han propiciado la exportación de textiles de esta naturaleza, con gran suceso.

En la fase de comercialización de la cadena productiva, se encuentran aquellos comerciantes orientados al mercado interno y otros al mercado externo.

En el Perú, la comercialización en el mercado interno, de los productos textiles en base a lana de oveja, está asociada al turismo; compiten en este aspecto con los textiles provenientes de los camélidos andinos. Puntos importantes se ubican las ciudades de Cusco, Lima, Puno, Arequipa, Huancayo y Huaraz, precisamente por constituir grandes centros de atracción turística.

A nivel de exportaciones, los productos en base a lana ovina se orientan principalmente a la artesanía.
La distribución de la producción de ganado ovino en el Perú se muestra en el Gráfico N. ${ }^{\circ} 1$, según el cual el mayor productor de ovinos es Puno, seguido de Cusco, Junín y Huánuco.

El ganado ovino en el Perú está conformado por 90 $\%$ de ganado criollo, el cual proviene del ganado ovino español cruzado con diversas razas como la Merino y la Corriedale, entre otras. El ganado ovino criollo fue mejorado y adaptado a condiciones adversas climáticas. Las características de rusticidad y resistencia son las más sobresalientes. Este ganado tiene buena conversión alimenticia. El ganado pastorea áreas pobres que sólo puede ser superada por la rusticidad de los camélidos. La producción de lana en los últimos 10 años no ha tenido variación significativa, lo cual está entre el rango de 10,000 a 13,000 toneladas de lana sucia y para el 2004 se reportó 11,674 toneladas. A nivel departamental Puno es el principal productor el $42.23 \%$ de la producción nacional, lo cual corresponde a 4,905 toneladas. Los precios de lana ovina se ven afectados por la caída de precios en el mercado mundial, desorganización de los productores y comercialización basada en lanas de calidad media de 24-28 micras y lanas gruesa mayor a 30 micras. El precio promedio de la lana sucia al productor nacional es de 0.80 centavos de dólar/lb. y el precio promedio de la lana limpia es 1-1.5 dólar/Lb. Las exportaciones de lana vienen creciendo en el rubro de bolas Top's, 
Perú para el año 2005, logró en $\$ 1,700,478.21$ a la fecha, siendo los países con destino de exportación Italia, Alemania, China, Ecuador, Irán, Japón, Suiza, Reino Unido. (Díaz, Rosario, 2007).

El número de empresas de textiles y confecciones en el Perú se muestra en el Cuadro 3.

\section{CUADRO 3. NÚMERO DE EMPRESAS SEGÚN ACTIVIDAD}

\begin{tabular}{|l|c|c|c|}
\hline Departamento & $\begin{array}{c}\text { Hilatura } \\
\text { yejeduría }\end{array}$ & $\begin{array}{c}\text { Acabados } \\
\text { textiles }\end{array}$ & $\begin{array}{c}\text { Confecciones } \\
\text { textiles }\end{array}$ \\
\hline Lima & 898 & 1418 & 1578 \\
\hline Cusco & 71 & 23 & 118 \\
\hline Puno & 133 & 49 & 126 \\
\hline Huánuco & 6 & 15 & 27 \\
\hline Huancavelica & 26 & 6 & 5 \\
\hline Ancash & 12 & 15 & 38 \\
\hline Cajamarca & 4 & 5 & 26 \\
\hline Huancavelica & 26 & 6 & 5 \\
\hline Otros & 323 & 468 & 805 \\
\hline Total & 1499 & 2005 & 2728 \\
\hline
\end{tabular}

Fuente: Unidad de Estadística Ministerio de la Producción.

Según este cuadro, hay una pronunciada concentración de establecimientos en Lima, seguidos de Puno, Cusco y Huancavelica. Puede observarse que no hay correspondencia entre los departamentos con producción significativa de ganado ovino y el número de empresas.

\section{ANÁLISIS Y DISCUSIÓN}

En el ámbito de los textiles y confecciones, el Perú presenta como producto superlativo el algodón y sus derivados, aspectos en donde el Ministerio de Agricultura viene desarrollando y fortaleciendo la correspondiente cadena productiva. Entre los productos textiles artesanales, las lanas de alpaca y vicuña son las que tienen una presencia significativa tanto en el mercado nacional como en las exportaciones. La lana de oveja está bastante relegada.

Algunas empresas privadas vienen apostando por este tipo de fibras naturales, tratando de enlazar los diferentes eslabones de la cadena, tal es el caso de SURITEC S.A.C en Huancayo, y otras similares en el sur peruano. Sin embargo, un importante número de productores de ovinos son pequeños propietarios, con orientación al autoconsumo y escasos criterios de calidad.
En cada una de las fases de la cadena productiva se presentan limitaciones. En la fase primaria, una limitante es la falta de criterio técnico para seleccionar las ovejas según el propósito del negocio (carne o lana), acompañado en muchas zonas andinas por prejuicios de orden cultural. En la fase industrial, no toda la lana acopiada pasa por las exigencias que requiere la industria textil para obtener productos de calidad, una gran proporción de productores trabaja en la lógica del autoconsumo. Todo ello se refleja en la escasa presencia de los productos derivados de la lana de oveja en las exportaciones textiles, reflejando también las debilidades de los comercializadores, que alejados de los actores de las otras fases no logran presentar productos que tengan acogida.

Promover la asociatividad, inclusión de tecnología moderna en toda la cadena productiva y localización de nichos de mercado, como el de los productos naturales, pueden contribuir a mejores logros en este sector. Todo ello dentro de una perspectiva sistémica.

\section{CONCLUSIONES}

1. El Perú tiene tradición de producción ovina, pero la mayor proporción la constituyen la raza "criolla", sin embargo, no existe una estrategia que la canalice en un mayor nivel de desarrollo de la cadena productiva.

2. La lana de oveja tiene un reducido peso en las exportaciones textiles.

3. En el mundo hay una demanda creciente de productos orgánicos o naturales, que pueden ser canalizados por los diferentes componentes de la cadena productiva de la lana de oveja.

4. Los productos con fibras naturales (fibras y tintes) tienen demanda creciente.

5. Las ONG vienen desarrollando en diferentes regiones del país proyectos para promover empresas comunales o asociativas que cubre la cadena productiva de la lana de oveja a nivel de artesanía y productos básicos.

6. Es necesario localizar mayor número de empresas textiles y de confecciones en aquellas zonas de mayor producción de ovinos, tales como Puno, Cusco, Huancavelica, Huánuco, entre otros.

7. La cadena productiva de la lana de oveja presenta serias limitaciones en las diferentes fases de la misma. Urge una visión integral del proceso y mayor coordinación entre los actores que la componen. 


\section{REFERENCIAS BIBLIOGRÁFICAS}

COFECYT (2008). Debilidades y desafíos tecnológicos del sector productivo lácteo ovino. Argentina. http://www.cofecyt.mincyt.gov.ar/pcias_ pdfs/la_pampa/UIA_lacteo_ovino_08.pdf. (Visitado en noviembre 2009).

Díaz, R. (2007). Sector ovinos en el Perú con perspectivas al 2015. V Congreso de Especialistas en Pequeños Rumiantes y Camélidos Sudamericanos, Mendoza, Argentina.

FAO. Hojas informativas año internacional de las fibras naturales 2009. http://www.naturalfibres2009.org/es/aifn/recursos.html, (Visitado en noviembre 2009).

Gomes de Castro, A. (2002). Cadena productiva: Marco conceptual para apoyar la prospección tecnológica. Revista Espacios Vol 23 N. ${ }^{\circ} 2$.

Huamán, W. (2003). Ingeniería en la capacitación de operarios para la industria de la confección textil. Tesis para optar el Título de Ingeniero Industrial. FII, UNMSM.
Ministerio de Agricultura (2009). Cadenas productivas. http://www.minagricultura.gov.co/02 componentes/06com_01b_cadenas.aspx

Ministerio de Agricultura Perú (2009) Unidad de Estadística. Consultado en: http://www.minag.gob. pe/estadisticas/dinamica-agropecuaria.html (Visitado en octubre 2009).

Ministerio de la Producción Perú (2009) Unidad de Estadística. Boletines mensuales. http://www. produce.gob.pe/portal/portal/apsportalproduce/ internaproduce?ARE $=1 \& \mathrm{JER}=20$

Proyecto Desarrollo Corredor Cusco-Puno (2006). Fibra de lana y alpaca.

San Martín, F. (2008). Territorios y empresas en Red. Organización MINKA PERÚ.

Tecnología Confección Textil. (2008). Las fibras textiles. Capítulo 3. Consultado en: http://www. detextiles.com/files/cap_03.pdf 